\title{
The Morphology and Ultrastructure of the Spore and Exosporium of Some Clostridium Species
}

\author{
BY W. HODGKISS, Z. J. ORDAL AND D. C. CANN \\ Ministry of Technology, Torry Research Station, Aberdeen
}

(Accepted for publication 10 December 1966)

\begin{abstract}
SUMMARY
The spores of original isolates of Clostridium species obtained from fresh and processed marine fish and from marine bottom deposits were compared with the spores of culture collection strains of the same species. These strains were Clostridium botulinum type E, C. sporogenes, $C$. bifermentans and $C$. sordellii. Spores of unclassified, non-toxigenic isolates which resemble $C$. botulinum type $\mathrm{E}$, of ' $\mathrm{OS}$ mutants', of culture collection strains of $C$. botulinum types $\mathrm{A}$ and $B$ and an isolate of type $F$ were also examined. The gross morphology and ultrastructure of the spores and exosporia were studied in electron microscopic preparations by using the techniques of metal shadowing, carbon replica, negative-staining and ultra-thin sectioning. Spores of some strains were found to possess tubular appendages. The morphology of the spores and the ultrastructural detail of the spore appendages and exosporia are described.
\end{abstract}

\section{INTRODUCTION}

Information on the fine structure of the spores of Clostridium species is limited. Franklin \& Bradley (1957) used the carbon replica technique to study the surface structure of the spore of certain Bacillus species and of $C$. welchii. Most of the electron microscopic studies on clostridial spores have been carried out on ultra-thin sections. Hashimoto \& Naylor (1958) used stained preparations for light microscopy, and ultrathin sections for electron microscopy, in studies of the sporulation process and the structure of the spores of $C$. sporogenes. The processes of sporulation and germination in C. butyricum and C. botulinum type E were studied by Takagi, Kawata \& Yamamoto (1960) and in C. tetani and C. histolyticum by Takagi, Kawata, Yamamoto, Kubo \& Okita (1960) using ultra-thin sections. This technique was also used by Fitz-James (1962) to follow spore development in $C$. pectinovorum. Takagi, Nakamura \& Ueda (1965) used both ultra-thin sections and the negative-staining technique to study the role of the intracytoplasmic membrane system during sporulation in C. tetani and C. botulinum type $\mathrm{E}$. In a comprehensive study of sporulation and spore germination in strain NCA62A (ATCC7948) of C. botulinum type A, Stewart (1963) used ultra-thin section and metal-shadowing techniques.

Walker (1963), in a study of spore antigens, published micrographs of metalshadowed entire spores of $C$. sporogenes and $C$. bifermentans which showed the presence of distinct exosporia on the spores of these species. Krassil'nikov, Duda \& Sokolov (1964) presented micrographs of metal-shadowed entire spores of 20 soil isolates of Clostridium species, demonstrating that these spores possessed elaborate and varied surface protrusions. Subsequently, Hodgkiss \& Ordal (1966), using metal-shadowing, 
negative-staining and carbon replica techniques, showed that the spore of $C$. botulinum type E possesses unusual tubular appendages and a delicate exosporium. Hodgkiss, Ordal \& Cann (1966) then carried out a comparative study of the morphology and ultrastructure of the spore and exosporium of $C$. botulinum type $\mathrm{E}$ and of the 'opaque sporulating (OS) mutants' of Dolman (1957).

These latter studies have been extended to investigate the morphology and ultrastructure of the spores of representative strains of certain Clostridium species isolated during surveys of the incidence of $C$. botulinum type $\mathrm{E}$ in fresh and processed marine fish and marine mud (Hobbs, Cann, Wilson \& Shewan, 1965; Cann et al. 1965; Cann, Wilson, Shewan \& Hobbs, 1966). For comparison, some Culture Collection strains of Clostridium species have also been studied.

\section{METHODS}

The spores of 38 strains of Clostridium species have been examined. The details of the strains are as follows:

$$
\begin{aligned}
& \text { TRS }=\text { Torry Research Station. } \\
& \text { NCIB }=\text { National Collection of Industrial Bacteria. } \\
& \text { NCTC }=\text { National Collection of Type Cultures. } \\
& \text { ATCC }=\text { American Type Culture Collection. } \\
& \text { FDA }=\text { Food and Drug Administration, U.S.A. }
\end{aligned}
$$

Strains of C. botulinum type $E$.

Strain 211: isolated from a sludge sample, Lake Abashiri, Japan (Nakamura et al. 1956).

$\left.\begin{array}{l}\text { NCIB } 4288 \\ \text { NCIB } 4299\end{array}\right\}$ TRS isolates from sea-bottom deposits, Scandinavian coast.

FT 4: TRS isolate from Norwegian herring (Clupea harengus)

NCIB 4248: Beluga strain. Isolated from 'muktuk', i.e. flippers of beluga (white whale, Delphinapterus leucas), Dolman \& Chang (1953).

Unclassified non-toxigenic (NT) strains which culturally and biochemically resemble C. botulinum type $E$

(a) NCIB 4266: TRS isolate from vacuum-packed, smoked haddock (Gadus aeglefinus). NCIB 4289: TRS isolate from sea-bottom deposit, Scandinavian coast. NCIB 4271: TRS isolate from cockles (Mytilus sp.), South Wales.

(b) NCIB 4268 ) NCIB 4269 NCIB 4270 NCIB 4277)

FT 3: TRS isolate from Norwegian herring (Clupea harengus).

OS strains (Dolman, 1957; Hobbs, Roberts \& Walker, 1965).

TRSEOs: TRS isolate from a culture of NCTC8266, C. botulinum type E. AJMAL os: isolated by M. Ajmal, Central Public Health Laboratory, Colindale, from a culture of a FDA tuna strain of $C$. botulinum type $\mathrm{E}$.

24 AS: isolated from vacuum-packed kippers (i.e. smoked herring, Clupea harengus). 
Strains of C. sporogenes

FT 23: isolated from vacuum-packed smoked haddock, (Gadus aeglefinus).

NCIB 532

NCIB 9383

PA 3679 (= ATCC 7955, NCIB 8053): Putrefactive anaerobe.

Strains of $C$. bifermentans

244B

247 B TRS isolates from vacuum-packed smoked haddock and kippers. $275 \mathrm{~B}$ $288 \mathrm{~B}$

NCIB 506, 1341, 2929, 6800, 6928.

Strains of C. sordellii

35 B: TRS isolate from vacuum-packed kippers.

NCIB 2914, 6801 and 6929.

CN1734: Burroughs Wellcome Culture Collection, Beckenham, England.

C. botulinum type $A$.

NCTC 7272

zK 3: Unilever Research Laboratories, Colworth House, Sharnbrook, Bedford, England (isolated from groundnuts, Arachis hypogea).

C. botulinum type $B$

ATCC7949

C. botulinum type $F$

610в: Craig \& Pilcher, (1966).

Spore suspensions.

Spore crops of the organisms were produced in a variety of nutrient media including Hartley's digest broth, Robertson's meat broth, blood-agar, egg-yolk agar (Willis \& Hobbs, 1958) and in the medium of Schmidt, Nank \& Lechowich (1962) or in that medium with added manganese and calcium. Spores were harvested, washed by lowspeed centrifugation $(2000 \mathrm{~g})$ and resuspended in $0.85 \%$ sodium chloride solution. The saline spore suspensions were then examined in the light microscope by phase contrast. If it was found that sporulation was not complete, vegetative debris was removed by treating the saline suspension with trypsin for $12-24 \mathrm{hr}$.

\section{Electron microscopy.}

The spores were further washed three times by low speed centrifuging $(2000 \mathrm{~g})$ in freshly distilled, sterile water. Droplets of this distilled water suspension were placed on formvar coated 200-mesh copper grids, air-dried and shadowed with gold + palladium $(40+60)$ at an angle of $20^{\circ}$. Carbon-coated 200-mesh copper grids were used for negative stained preparations. A droplet of the spore suspension was allowed to air-dry on the grid. The grid was then treated for $1 \mathrm{~min}$. with $1 \%$ aqueous ammonium molybdate or $1 \%$ aqueous phosphotungstic acid (PTA) adjusted to 
pH 7.0 (Brenner \& Horne, 1959) Carbon replicas were prepared by the method of Bradley \& Williams (1957) and were shadowed with gold + palladium $(40+60)$ at an angle of $20^{\circ}$. For sectioning, spores were fixed in $2.5 \%$ glutaraldehyde in $0.1 \mathrm{M}$-phosphate buffer ( $\mathrm{pH} \mathrm{7.0)}$ for $4 \mathrm{hr}$ at $4^{\circ}$, post-fixed in osmium tetroxide in veronal buffer for $2 \mathrm{hr}$ at $4^{\circ}$ and stained in $1 \%(\mathrm{w} / \mathrm{v})$ aqueous uranyl acetate for $18 \mathrm{hr}$ at $4^{\circ}$. The fixed and stained spores were then dehydrated by passage through increasing strengths of ethyl alcohol, transferred to propylene oxide, thence to $50+50(\mathrm{v} / \mathrm{v})$ propylene oxide and Epon and finally embedded in pure Epon (Luft, 1961). Ultra-thin sections were cut on a Cambridge Huxley Microtome using glass knives.

Preparations were examined in a Siemens Elmiskop I electron microscope using single- or double-condenser illumination, a $200 \mu$ condenser aperture, a $50 \mu$ objective aperture and accelerating voltages of $60 \mathrm{kV}$ (for sections and metal-shadowed preparations) or $80 \mathrm{kV}$. Micrographs were recorded at initial magnifications of $\times 8000$ to $\times 40,000$ on Ilford $\mathrm{N} 50$ plates.

\section{RESULTS}

During the surveys carried out in this laboratory (Hobbs, Cann, Wilson \& Shewan, 1965; Cann et al. 1965; Cann et al. 1966), some isolates identified as Clostridium sporogenes were obtained. The morphology of the spores of one of these strains, FT 23, was compared with that of the spores of two Culture Collection strains of C. sporogenes, NCIB 532 and 9383, and that of the spores of the well-documented strain commonly referred to as Putrefactive Anaerobe PA3679 (ATCC7955, NCIB 8053). The fine structure of the spores of all four strains was essentially identical (Pl. 1, fig. 1). The spore here appears as an electron-dense, ovoid body loosely enveloped in an exosporium, typical of what might be termed an orthodox bacterial endospore as described previously in many species (review by Robinow, 1960). The spore of $C$. sporogenes has a relatively smooth coat ( $\mathrm{Pl}$. 1, fig. 2), being devoid of surface ornamentation of the type found on the spores of certain Bacillus species (Hooff \& Aninga, 1956; Bradley \& Williams, 1957; Franklin \& Bradley, 1957; Dondero \& Holbert, 1957; Bradley \& Franklin, 1958) and devoid of protrusions or appendages (Krassil'nikov et al. 1964; Hodgkiss \& Ordal, 1966; Hodgkiss et al. 1966). The exosporium of the four strains of $C$. sporogenes may, for the purposes of this paper, be categorized as 'thick' as opposed to the thin, membranous structure which envelopes the spore of C. botulinum type E (Hodgkiss \& Ordal, 1966). In metal-shadowed preparations it measures approximately $120-150 \AA$ thick. No ultrastructural detail was discernible in micrographs of metal-shadowed or negatively stained preparations. This type of exosporium will therefore herein be described as homogeneous.

Included in the present study were four toxigenic strains of Clostridium botulinum type E which we had not examined previously (NCIB4288, 4299, Nakamura 211 and FT4). The spores of all strains showed the same morphology and ultrastructure as that found in the Beluga strain, NCrB 4248 (Hodgkiss \& Ordal, 1966), and the FDA tuna strain (Hodgkiss et al. 1966). The outstanding features of these spores are the presence of numerous tubular appendages (160-240) per spore and the delicate fibrillar ultrastructure of the membranous and large exosporium. With most strains the appendages appeared to be uniformly distributed over the spore surface as in the Beluga strain. However, in some strains the appendages appeared to be most numerous at the poles of the spore, e.g. FDA tuna strain (Hodgkiss et al. 1966). 
A number of isolates were obtained which were non-toxigenic (NT) but which in other characteristics closely resembled Clostridium botulinum type E. These NT cultures could be divided into two groups by spore morphology. First, there were those strains (NCIB 4266, 4271 and 4289) in which the morphology and ultrastructure of the spore and exosporium were identical with that of toxigenic strains of $C$. botulinum type E (Pl. 1, fig. 3). The spores of the second group of NT isolates (NCIB 4268, 4270, 4277, 4269 and FT3) were all similar to each other in morphology but were quite different from the first group just described. The spores were devoid of appendages and were enveloped in a thick, homogeneous exosporium (P1. 1, fig. 4).

Non-toxigenic 'mutants' of Clostridium botulinum type $\mathrm{E}$ which produce opaque sporulating (OS) colonies were described by Dolman (1957). Following the detailed studies of Hobbs, Roberts \& Walker (1965) in which three of the isolates (E60s, E70s and E1040s) were compared to a number of toxigenic strains of $C$. botulinum type E, we examined and reported on the spore morphology of the same three strains (Hodgkiss et al. 1966).) Since then we have examined the spores of three more strains (TRSEOS, AJMAL OS and 24AS). These possessed the same morphology and ultrastructure as the three already reported on. For comparative purposes we include a typical electron micrograph of a spore of strain $24 \mathrm{AS}$ (PI. 1, fig. 5) and will briefly summarize the pertinent characteristics. Each spore possesses from one to four openended tubular appendages which normally arise from the poles of the spore. The appendages vary in length (usually $1-2 \mu$ ) but are of relatively constant diameter. The overall diameter of the tubule is $480 \AA$ and that of the lumen $320 \AA$. Negatively stained preparations of the exosporium show a hexagonal pattern of holes, the centre to centre distance of these holes is approximately $90 \AA$ (Pl. 1, fig. 6).

The spores of the several Clostridium bifermentans strains were of two types. The spores of the five Culture Collection strains were of orthodox appearance; but the spores of the four strains (244 B, 247 B, 275 B and 288 B) isolated from vacuum-packed smoked haddock and kippers were striking and distinctive in morphology possessing appendages (usually from 10 to 20 ) which projected from the poles of the spore (Pl. 1, fig. 7; Pl. 2, fig. 8). These were approximately $2 \mu$ in length and the distal portion of the appendage was thicker than the proximal portion. Negatively stained preparations demonstrated the tubular nature and the distinctive fine structure of these appendages ( $\mathrm{Pl}$. 2, fig. 9). The overall diameter of the proximal portion of the tubule is $480 \AA$ and that of the lumen $240 \AA$. The distal portion of the tubule wall is thickened by an outer sheath which is composed of microfibrils, about $500 \AA$ in length and $25-40 \AA$ in diameter, arranged in a parallel array and inserted into the tubule wall at an angle of $40^{\circ}$ to the long axis of the tubule. In this respect they resemble the manner in which the barbs are inserted on the calamus of a bird's feather. The sheath of microfibrils thickens the distal portion of the tubule wall and thus increases the overall diameter of the appendage to the order of $1120 \AA$. The lumen of the tubule is of constant diameter throughout the entire proximal portion and along most of the thicker, distal portion of the appendage. However, at the extreme distal end the diameter increases gradually and the lumen terminates in a funnel-shaped orifice. The exosporium is of the thick homogeneous type. Suggestive evidence that the appendages arise from the spore coat is supplied in P1. 2, fig. 10. The short shadow of this spore indicates that the spore contents have been lost, but the appendages are still in their normal position. Further evidence that the appendages are extensions of the spore coat is presented below. 
A typical spore of one of the five Culture Collection strains of Clostridium bifermentans is presented in P1. 2, fig. 11. No appendages are present and the thick exosporium is of the homogenous type.

The five strains of Clostridium sordellii had spores with three types of morphology. Spores of strains NCIB 2914 and CN 1734 possessed a thick, homogeneous exosporium and lacked appendages (Pl. 2, fig. 12). Spores of strain 35 B (Pl. 2, fig. 13) had unusually long (up to $4 \mu$ ) open-ended tubular appendages which in many respects were similar to the appendages found on the OS spores, the overall diameter of the tubule ( $600 \AA)$ and the lumen diameter $(400 \AA)$ being slightly greater than that of the OS strains. They were commonly seen as a coiled structure within the disintegrating fine-meshed fibrillar exosporium (Pl. 2, figs. 14, 15). The exosporium appeared to be even more delicate than that of the type $\mathrm{E}$ strains, the individual fibrils were comparable in diameter $(60 \AA)$ to those of the type $E$ exosporium and their arrangement indicated a more loosely formed meshwork structure.

The spores of strain 6929, Colstridium sordellii (P1. 3, fig. 16) possessed simple openended tubular appendages similar in dimensions to those of strain 35 в but the exosporium was quite different. The exosporium was similar to that of strains NCIB 2914 and $\mathrm{CN} 1734$, i.e. it was of the 'thick', homogeneous type (PI. 3, fig. 17).

Because of our original interest in the spores of Clostridium botulinum type $\mathrm{E}$, we examined the spores of representative strains of types A, B and F. The strains we studied sporulated poorly in the media tested, and so our findings on these strains must be considered to be of a preliminary nature. A typical type-A spore as found in our preparations is presented in Pl. 3, fig. 18. Although the spore preparations appeared to be 'clean' as viewed by dark-phase contrast optics at a magnification of $\times 1250$, most spores seen in the electron microscope showed the tenacious attachment of sporangial fragments. Similar results were obtained with the strain of type B (ATCC 7949) although occasionally we did find a free spore (P1. 3, fig. 19). The spores of both the type-A and type-B strains were devoid of appendages. With both strains, when the cell sporulates, the sporangium assumes an almost diamond shape and the spore is located in the more obtuse end of the cell. When the spore reaches maturity, the tip of the sporangium furthest away from the spore begins to lyse, and lysis continues slowly toward the end containing the spore (Pl. 3, fig. 20-22). While several sporulating cultures were incubated for longer periods of time before harvesting and cleaning, lysis of the sporangial fragments adhering to the spore was not further improved.

A spore suspension of Clostridium botulinum type A, strain ZK 3, prepared by Dr A. C. Baird-Parker, showed the same morphological characteristics as did the preparations of strain NCTC 7272 (Pl. 3, fig. 23).

Spores of Clostridium botulinum type $\mathrm{F}$ were devoid of appendages but were enveloped in a distinct and voluminous, thick exosporium (P1. 4, fig. 24). Negatively stained preparations of the exosporium showed an ultrastructure with a definite hexagonal pattern similar to that found in the exosporium of the OS strains (PI. 4, fig. 25). The centre-to-centre distance of the hexagonal units was approximately $100 \AA$.

In addition to this survey of the morphological characteristics of some clostridial spores we have also initiated investigations to further describe the attachment of the appendages to the spore and ultimately to follow their development during sporulation and their fate during spore germination. While this effort is still in its initial stages we consider it pertinent to present some of our findings on the Beluga strain of 
C. botulinum type E (NCIB 4248). The appearance of an ultra-thin section of a liberated spore is presented in $\mathrm{Pl} .4$, fig. 26. The spore proper consists of a relatively dense core surrounded by a less-dense cortex with a multi-layered spore coat (cf. Robinow, 1960). The thin, inner layer of the spore coat is very electron-dense but the outer layers are less dense. The tubular appendages appear to be extensions of the outer spore coats as they do not penetrate through the coat into the core or cortex. The whole of the
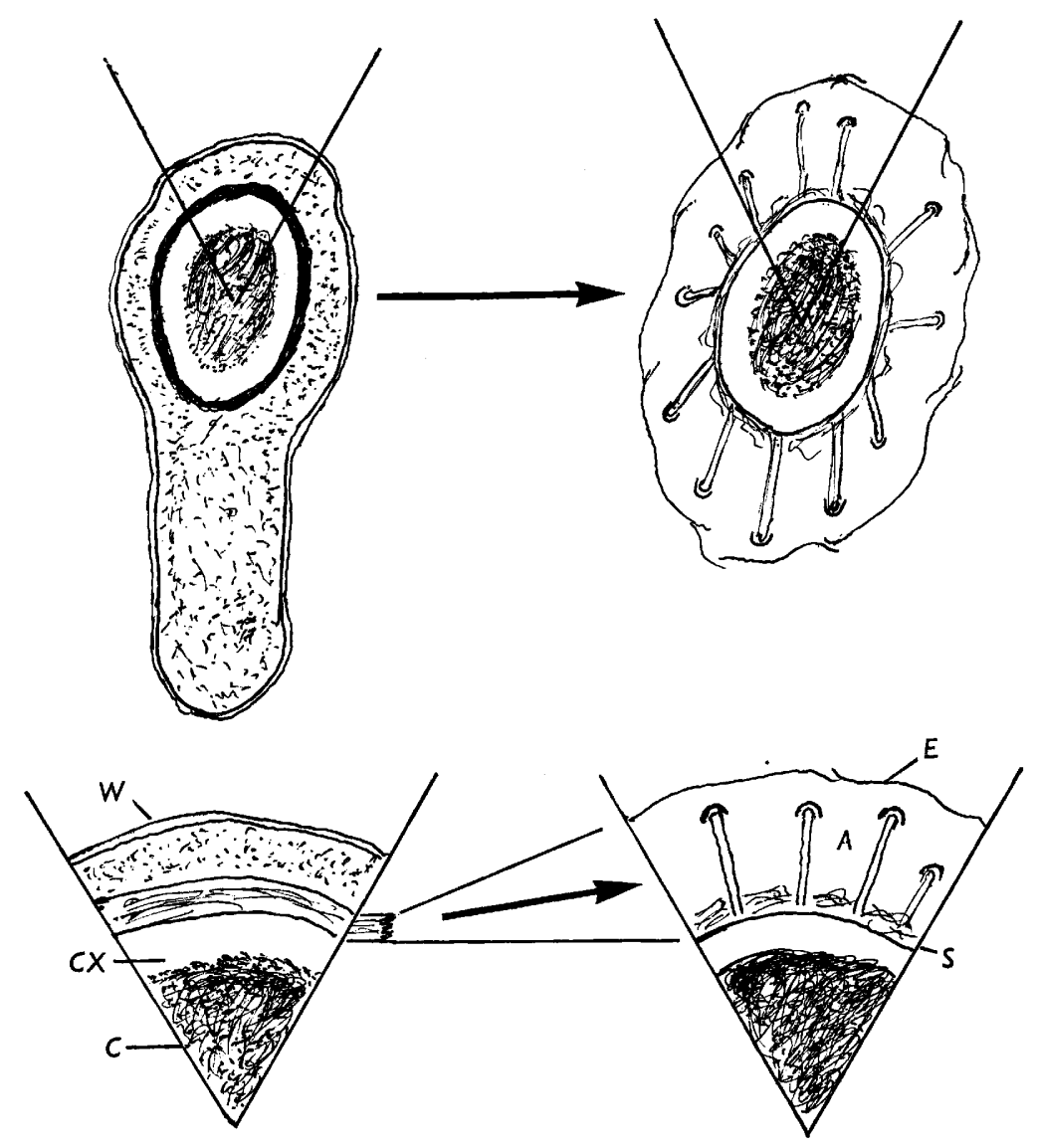

Fig. 1. Clostridium botulinum type E. A diagrammatic representation of the appearance, in ultra-thin sections, of a developing spore in a sporangium and a mature, liberated spore within its exosporium, $C=$ core, $C X=$ cortex, $W=$ wall of sporangium, $S=$ spore coat, $A=$ appendages, $E=$ exosporium.

spore is encased in a thin voluminous exosporium which is composed of microfibrils (Hodgkiss \& Ordal, 1966). The relative size of the complete liberated spore in its exosporium and that of a normal vegetative cell is presented in Pl. 4, fig. 27. Our observations indicate that whilst the spore is within the sporangium the appendages and exosporium are tightly wrapped around the spore. When the mature spore is liberated from the sporangium, fluid is absorbed through the exosporium causing it to become turgid and swollen. During this process the appendages unfold and become everted. These events are diagrammatically represented in Fig. 1. 


\section{DISCUSSION}

Our findings of varied and elaborate appendages on the spores of certain strains substantiate those of Krassil'nikov et al. (1964), who described elaborate protrusions on the surface of spores of Clostridium species. On the basis of their studies these authors consider that, in this genus, spore morphology is a species-specific characteristic. Our studies were carried out on a very narrow range of species so that our results can be discussed only within this limitation.

Morphologically the spores of the strains studied may be divided into two main groups: those with appendages; and those without-the orthodox spores. The appendage-bearing spores may be further characterized on the basis of the ultrastructure of the appendages. The constant and outstanding feature of the appendages is that they are micro-tubules. Those of the spores of $C$. botulinum type $\mathrm{E}$ are sealed at the distal end (Hodgkiss \& Ordal, 1966) but in the other strains the distal end of the tubule is open. Again, in the type-E strains the tubules remain within the intact exosporium, whereas in the other strains the tubules eventually protrude through the fractured exosporium.

In all except three of the strains (Clostridium botulinum type A, NCTC7272; ZK 3 and type B, ATCC7949) the spore was enveloped in an exosporium. Three main structural types of exosporium were found: a thin membranous type composed of microfibrillar elements, a thick type in which a hexagonal pattern may be detected and a thick homogeneous type.

The above, then, are the points of morphological differentiation of the spore and exosporium. The orthodox or plain spores could not be further differentiated morphologically as they were all devoid of the type of surface ornamentation found in some Bacillus species and all strains showed variation in spore size (see also Robinow, 1960).

In addition to four strains of Clostridium botulinum type $E$ listed above, five other strains have previously been examined (Hodgkiss \& Ordal, 1966; Hodgkiss et al. 1966). All the strains were found to have the same morphology of spore appendages and exosporium.

The relationship between the unclassified NT strains and strains of Clostridium botulinum type $\mathrm{E}$ is not clear. Repeated cultural and biochemical tests fail to distinguish between the two. Similar strains have been isolated, along with type-E strains, by Dolman, Darby \& Lane (1955) from post-mortem stomach contents in a case of botulism and by Nakamura et al. (1956) from three samples of 'Izushi', all of which had caused outbreaks of botulism. 'Izushi' is a food prepared in the coastal districts of Hokkaido. Raw fish patties mixed with cooked rice, diced vegetables, salt, vinegar and red pepper, and placed in wooden tubs under pressure, are allowed to ferment for 3-4 weeks. Details of the preparation are given by Nakamura et al. (1956). Chapman \& Naylor (1966) isolated two similar NT strains, as well as two type-E strains, from Cayuga Lake fish during a survey of the incidence of type $\mathrm{E}$ in lake water and in fish. Kautter, Harmon, Lynt \& Lilly (1966) and Bott, Defner, McCoy \& Foster (1966) have also isolated NT strains from fish from the Great Lakes.

The serological relationships of different strains of Clostridium botulinum type $\mathrm{E}$ are complex (Nakamura et al. 1956). It is not surprising therefore that serological tests in this laboratory have failed to provide a key to the relationship of the NT strains to strains of $C$. botulinum type E. However, in view of the uniformity of spore morpho- 
logy in type-E strains it is tempting to speculate that the three NT strains which possess spores of exactly this type are non-toxigenic mutants of type E. In the case of those NT strains which possess orthodox spores, spore morphology would not itself suggest a close relationship to type $\mathrm{E}$.

Studies on three additional OS strains have substantiated our original findings on Dolman's strains (Hodgkiss et al. 1966). In all these OS strains there is uniformity of spore morphology and of appendage and exosporium ultrastructure. The gross morphology of the OS spore resembles that of the spore of Clostridium sporosetum sp.nov. as described by Krassil'nikov et al. (1964). In its ultrastructure the exosporium of the OS strains is similar to that of Bacillus cereus (Gerhardt \& Ribi, 1964). It is our view that the marked differences in spore morphology between the type $E$ strains and the OS strains would not point to a close relationship between the two (Dolman, 1957). Rather, it would seem to be evidence to support the views of Muriel Robertson (quoted on p. 56 by Dolman, 1964) and to add weight to the contention of Hobbs et al. (1965) that the OS strains are commensal organisms.

The speciation of organisms which belong to the Clostridium bifermentans $-C$. sordellii group is complex and has been the subject of some controversy (Brooks \& Epps, 1959; Huang, 1959, Walker, 1963; Nishida, Tamai \& Yamagishi, 1964; Tamai \& Nishida, 1964; Huang, Tamai \& Nishida, 1965). Within this group of organisms we have demonstrated that strains with very different spore morphology are to be found. The morphological characteristics of the four fish isolates of $C$. bifermentans were constant and contrasted sharply with the orthodox spores found in the five Culture Collection strains. These four strains were arbitrarily selected from approximately 100 such isolates which had been collected as representative clostridial strains found in marine fish products. Their cultural and biochemical reactions were typical of $C$. bifermentans as defined by Brooks \& Epps (1959). The gross morphology of the spores of these four strains resembles that of the spore of $C$. sporopenitum sp. nov. of Krassil'nikov et al. (1964). The biochemical reactions and ultrastructural details of the spore of this organism have not yet been published so we cannot make further comparison. Three morphological types of spores were found in the five strains which, according to the scheme of Brooks \& Epps (1959), could be classified as $C$. sordellii. The fish isolate, strain 35 B, and a Culture Collection strain, NCIв 6929, were similar in that they had spores upon which long, open-ended appendages were present but the exosporia of the two strains were quite different in ultrastructure. The other three Culture Collection strains had orthodox spores enveloped in homogeneous exosporia.

The micrographs of spores of Clostridium botulinum types A, B and F record our initial observations on these three toxin types. In our preparations the spores of all three types were very clearly differentiated morphologically from those of the type $E$ strains. We found no evidence of an exosporium in types A and B. For the two type-A strains (NCTC7272 and zK3) this finding is in contrast to the findings of Stewart (1963) who demonstrated the presence of an exosporium in strain NCA62A (ATCC 7948). The spore of the type-F strain is distinguishable from those of the strains of types A and B studied here by the presence of an exosporium which has a hexagonal-pattern ultrastructure.

The orthodox or plain type of spore with a homogeneous, thick exosporium was common to the four strains of Clostridium sporogenes, the five Culture Collection strains of $C$. bifermentans, three Culture Collection strains of $C$. sordellii and five of 
the NT strains. Walker (1963) also found this type of spore in strains of $C$. sporogenes, $C$. bifermentans and $C$. sordellii. The morphology of this type of spore is therefore of little aid, except as a negative feature, as a determinative tool. However, the elaborate spores described above, which are of consistent morphology, may well come to be regarded as a valuable aid in determinative and taxonomic studies. Clearly a wider range of species must be investigated to assess fully the value of spore morphology within the genus Clostridium.

Structures as elaborate as the clostridial appendages have not been described on spores of Bacillus nor on spores of Streptomyces species. In both these genera characteristic spore surface ornamentation has been described and the taxonomic significance thereof outlined (Bradley \& Franklin, 1958; Tresner, Davies \& Backus, 1961).

The origin, the function and the eventual fate of the microtubular appendages during spore germination have yet to be demonstrated and elucidated. Preliminary observations on ultrathin-sections of sporulating cells and mature spores indicate that the microtubular appendages are a part of the outer coats of the spore. Our studies show that during development of the spore within the sporangium the long appendages are tightly wrapped around the spore and are coiled up within the exosporium. Upon lysis of the sporangium with its concurrent freeing of the spore the exosporium absorbs fluid, enlarges in volume, and the appendages uncoil. With spores of the Clostridium botulinum type $\mathrm{E}$ the appendages are normally contained within the exosporium whereas in the other types that we have observed, the long tubules eventually break through the exosporium and protrude out into the surrounding medium. The consistent tubular nature of the varied appendages that we have described is probably the most interesting and puzzling aspect of the entire study. What is their function? At this stage of our studies nothing can be added to the conjecture previously advanced (Hodgkiss \& Ordal, 1966). Clearly this is a challenge for future studies on spore formation and germination.

We wish to thank Barbara B. Wilson for her co-operation in this study, Dr J. M. Craig for a culture of strain 610B, Dr Y. Nakamura for a culture of strain 211, Dr A. C. Baird-Parker for a spore suspension of strain $\mathrm{ZK} 3$ and $\mathrm{Mr} \mathrm{M}$. Ajmal for a culture of strain FDAOS.

During this investigation Z. J. Ordal was in receipt of a Public Health Fellowship 1-F3-A 1-28, 391-01, from the National Institute of Allergy and Infectious Diseases. The work described in this paper was done as part of the programme of the Ministry of Technology.

\section{REFERENCES}

BotT, T. L., Defner, J. S., McCoy, E. \& Foster, E. M. (1966). Clostridium botulinum type E in fish from the Great Lakes. J. Bact. 91, 919.

Bradley, D. E. \& Frankin. J. G. (1958). Electron microscope survey of the surface configuration of spores of the genus Bacillus. J. Bact. 76, 618.

BRADLEY, D. E. \& Williams, D. J. (1957). An electron microscope study of the spores of some species of the genus Bacillus using carbon replicas. J. gen. Microbiol. 17, 75.

Brenner, S. \& Horne, R. W. (1959). A negative staining method for high resolution electron microscopy of viruses. Boichim. biophys. Acta 34, 103.

Brooks, M. E. \& Epps, H. B. G. (1959). Taxonomic studies of the genus Clostridium: Clostridium bifermentans and C. sordellii. J. gen. Microbiol. 21, 144. 
CANN, D. C., Wilson, B. B., Shewan, J. M. \& HobBs, G. (1966). Incidence of Clostridium botulinum type $\mathrm{E}$ in fish products in the United Kingdom. Nature, Lond. 211, 205.

Cann, D. C., Wilson, B. B., Hobrs, G., Shewan, J. M. \& Johannsen, A. (1965). The incidence of Clostridium botulinum type $\mathrm{E}$ in fish and bottom deposits in the North Sea and off the coast of Scandinavia. J. appl. Bact. 28, 426.

Chapman, H. M. \& NAYLoR, H. B. (1966). Isolation of Clostridium botulinum type E from Cayuga Lake fish. Appl. Microbiol. 14, 301.

Craig, J. M. \& Pilcher, K. S. (1966), Clostridium botulinum type F: isolation from salmon from the Columbia River, Science, N.Y. 153, 311.

Dolman, C. E. (1957). Recent observations on type E botulism. Can. J. publ. Hlth 48, 187.

Dolman, C. E. (1964). Growth and metabolic activities of C. botulinum types. In Botulism, Proceedings of a Symposium. Publ. Hlth. Serv. Publs., Wash. no. 999-FP-1.

Dolman, C. E. \& Chang, H. (1953). The epidemiology and pathogenesis of type E and fish-borne botulism. Can. J. Publ. Hlth 44, 231.

Dolman, C. E., Darby, G. E. \& Lane, R. F. (1955). Type E botulism due to salmon eggs. Can. J. publ. Hlth 46, 135.

Dondero, N. C. \& Holbert, P. E. (1957). The endospore of Bacillus polymyxa. J. Bact. 74, 43.

FITZ-JAMES, P. C. (1962). Morphology of spore development in Clostridium pectinovorum. J. Bact. 84, 104.

Franklin, J. G. \& Bradley, D. E. (1957). A further study of the spores of the genus Bacillus in the electron microscope using carbon replicas, and some preliminary observations on Clostridium welchii. J. appl. Bact. 20, 467.

GerhaRDT, P. \& RIBI, E. (1964). Ultrastructure of the exosporium enveloping spores of Bacillus cereus. J. Bact. 88, 1774.

Hashimoto, T. \& NAYLOR, H. B. (1958). Studies on the fine structure of microorganisms. II. Electron microscopic studies of sporulation of Clostridium sporogenes. J. Bact. 75, 647.

HobBs, G., RobertS, T. A. \& WALkeR, P. D. (1965). Some observations on OS variants of Clostridium botulinum type E. J. appl. Bact. $28,147$.

Hobss, G., CanN, D. C., Wilson, B. B. \& Shewan, J. M. (1965). The incidence of organisms of the genus Clostridium in vacuum packed fish in the United Kingdom. J. appl. Bact. 28, 265.

Hodgkiss, W. \& Ordal, Z. J. (1966). The morphology of the spore of some strains of Clostridium botulinum type E. J. Bact. 91, 2031.

Hodgkiss, W., ORdal, Z. J. \& CANN, D. C. (1966). The comparative morphology of the spores of Clostridium botulinum type $\mathrm{E}$ and the spores of the 'OS mutant'. Can. J. Microbiol. $12,1283$.

VAN DEN HoOfF, A. \& ANINGA, S. (1956). An electron microscope study on the shape of spores of Bacillus polymyxa. Antonie van Leeuwenhoek 22, 327.

HuAng, C, T. (1959). Comparison of Clostridium bifermentans and Clostridium sordellii. Ph.D. Thesis, University of Leeds.

Huang, C. T., TAmai, K. \& Nishida, S. (1965). Taxonomy of Clostridium bifermentans and Clostridium sordelli. III. Agglutinability of heat-resistant sub-strains of Clostridium sordellii. J. Bact. $90,391$.

KautTer, D. A., HARmon, S. M., LyNT, R. K., JUN. \& Lilly, T. JUN. (1966). Antagonistic effect on Clostridium botulinum type E by organisms resembling it. Appl. Microbiol. 14, 616.

Krassil'nikov, N. A., Duda, V. I. \& Sokolov, A. A. (1964). Protrusions on the surface of spores of anaerobic bacteria of the genus Clostridium. Mikrobiologiya 33, 454.

LufT, J. (1961). Improvements in epoxy resin embedding method. J. biophys. biochem. Cytol. 9, 409.

Nakamura, Y., Iida, H., Saeki, K., Kanzawa, K. \& Karashimada, T. (1956). Type E botulism in Hokkaido, Japan. Jap. J. med. Sci. Biol. 9, 45.

Nishida, S., TAmaI, K. \& Yamagishi, T. (1964). Taxonomy of Clostridium bifermentans and Clostridium sordellii. I. Their toxicity, urease activity and sporulating potency. J. Bact. 88, 1641 .

Robinow, C. F. (1960). Morphology of bacterial spores, their development and germination. In The Bacteria. Ed. by I. C. Gunsalus and R. Y. Stanier, vol. r, p. 207. London: Academic Press.

SCHMIDT, C. F., NANK, W. K. \& LECHOWICH, R. V. (1962). Radiation sterilization of food. II. Some aspects of the growth, sporulation and radiation resistance of spores of Clostridium botulinum type E. J. Food Sci. $27,77$.

StEWART, G. J. (1963). Studies on spores of Clostridium botulinum. Ph.D. Thesis, West Virginia University, Morgantown.

TAKaGi, A., KaWATA, T. \& Yamamoto, S. (1960). Electron microscope studies on ultra-thin sections of spores of the Clostridium group, with special reference to the sporulation and germination process. J. Bact. 80, 37.

Takagi, A., Kawata, T., Yamamoto, T., Kubo, T. \& Okita, S. (1960). Electron microscope studies on ultra-thin sections of spores of Clostridium tetani and Clostridium histolyticum, with special reference to sporulation and spore germination process. Jap. J. Microbiol. 4, 137. 
TAKagi, A., NAKamuRA, K. \& UedA, M. (1965). Electron microscope studies of the intracytoplasmic membrane system in Clostridium tetani and Clostridium botulinum. Jap. J. Microbiol. 9, 131.

TAMAI, K. \& NishIDA, S. (1964). Taxonomy of Clostridium bifermentans and Clostridium sordellii. II. Toxigenic and sporulating potencies in substrains of a Clostridium sordellii strain. J. Bact. 88, 1647.

Tresner, H. D., DaVIEs, M. C. \& Backus, E. J. (1961). Electron microscopy of Streptomyces spore morphology and its role in species differentiation. J. Bact. 81, 70.

WALKER, P. D. (1963). The spore antigens of Clostridium sporogenes, $C$. bifermentans and $C$. sordellii. J. Path. Bact. 85, 41.

WIILIS, A. T. \& HoBBS, G. (1958). A medium for the identification of clostridia producing opalescence in egg yolk emulsions. J. Path. Bact. 75, 299.

\section{EXPLANATION OF PLATES}

Except where stated otherwise the electron-micrographs are of gold-palladium shadowed preparations and the graduation mark is $1 \cdot 0 \mu$.

\section{Plate 1}

Fig. 1. Clostridium sporogenes strain FT23, spore and exosporium.

Fig. 2. Putrefactive Anaerobe, PA 3679. Single stage carbon replica of a spore.

Fig. 3. NT strain NCIB 4271. The spore and its appendages are enclosed in a delicate, voluminous exosporium.

Fig. 4. NT strain NCIB 4270. Spores and exosporia.

Fig. 5. OS strain $24 \mathrm{AS}$. A spore with a single appendage protruding through the exosporium.

Fig. 6. OS strain 24AS. Ammonium molybdate preparation of exosporium showing a hexagonal pattern structure.

Fig. 7. C. bifermentans, strain $247 \mathrm{~B}$. The appendages of the spore are coiled up within the exosporium at one end and protrude through the ruptured exosporium at the other end.

\section{Plate 2}

Fig. 8. C. bifermentans, strain 288 в. Three spores showing the typical morphology.

Fig. 9. C. bifermentans, strain 288B. Negatively stained preparation showing the distal end of two appendages. The micro-fibrillar sheath and the funnel-shaped orifice of the tubule are demonstrated. Fig. 10. C. bifermentans, strain 288в. One spore is empty (arrow: note short shadow) and the appendages remain attached to the spore coat.

Fig. 11. C. bifermentans, strain NCrB 506. Spore and exosporium.

Fig. 12. C. sordellii, strain NCIB2914. Spores and exosporia.

Fig. 13. C. sordelli, strain 35 в. Note the delicate exosporium and the long, coiled appendages.

Fig. 14. C. sordellii, strain 35в. Ammonium molybdate preparation. The tubular nature of the appendages and the micro-fibrillar structure of the exosporium are demonstrated.

Fig. 15. C. sordellii, strain 35в. Appendages coiled within the exosporium in which micro-fibrillar structure is apparent.

\section{Plate 3}

Fig. 16. C. sordellii, strain NCIB6929. The spores possess long appendages and a homogeneous exosporium.

Fig. 17. C. sordellii, strain NCIB 6929. Ammonium molybdate preparation showing tubular appendages protruding through the ruptured, homogeneous exosporium.

Fig. 18. C. botulinum type A, strain NCTC7272. A spore within the remnants of the sporangium.

Fig. 19. C. botulinum type B, strain ATcc7949. A free spore.

Fig. 20 to Fig. 22. C. botulinum type A, strain NCTC7272. Three morphological forms seen in spore preparations. A wholly electron-dense sporangium, an almost entirely electron-dense diamond-shaped stage of the sporangium and the last stage in which the electron-dense spore is seen within the less electron-dense remnants of the sporangium.

Fig. 23. C. botulinum type A, strain $\mathrm{zx}$ 3. Typical field, spore preparation; the spores are seen within the remnants of sporangia. 
Journal of General Microbiology, Vol. 47, No. 2

Plate 1
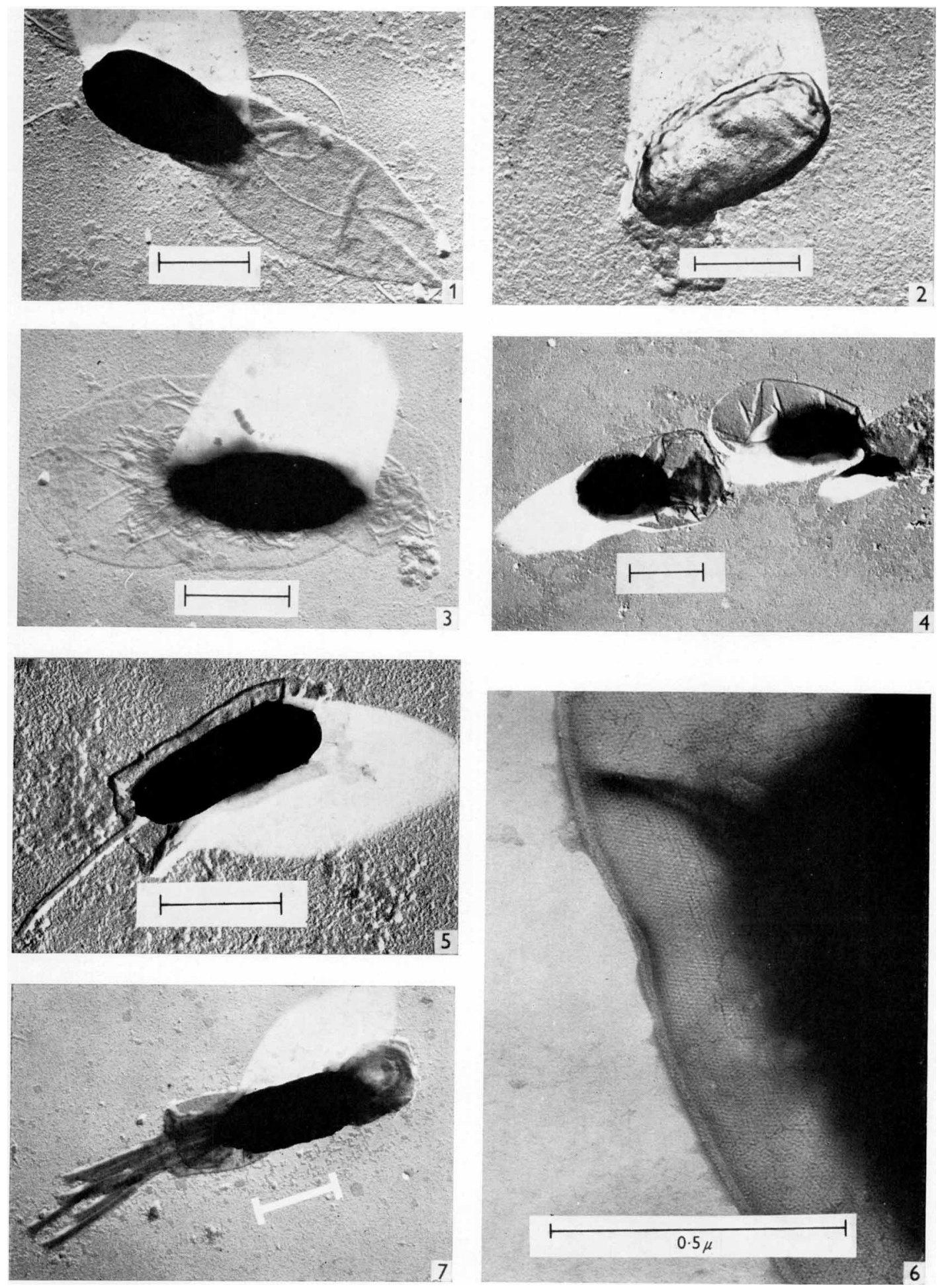

W. HODGKISS, Z. J. ORDAL AND D. C. CANN

(Facing p. 224) 
Journal of General Microbiology, Vol. 47, No. 2

Plate 2
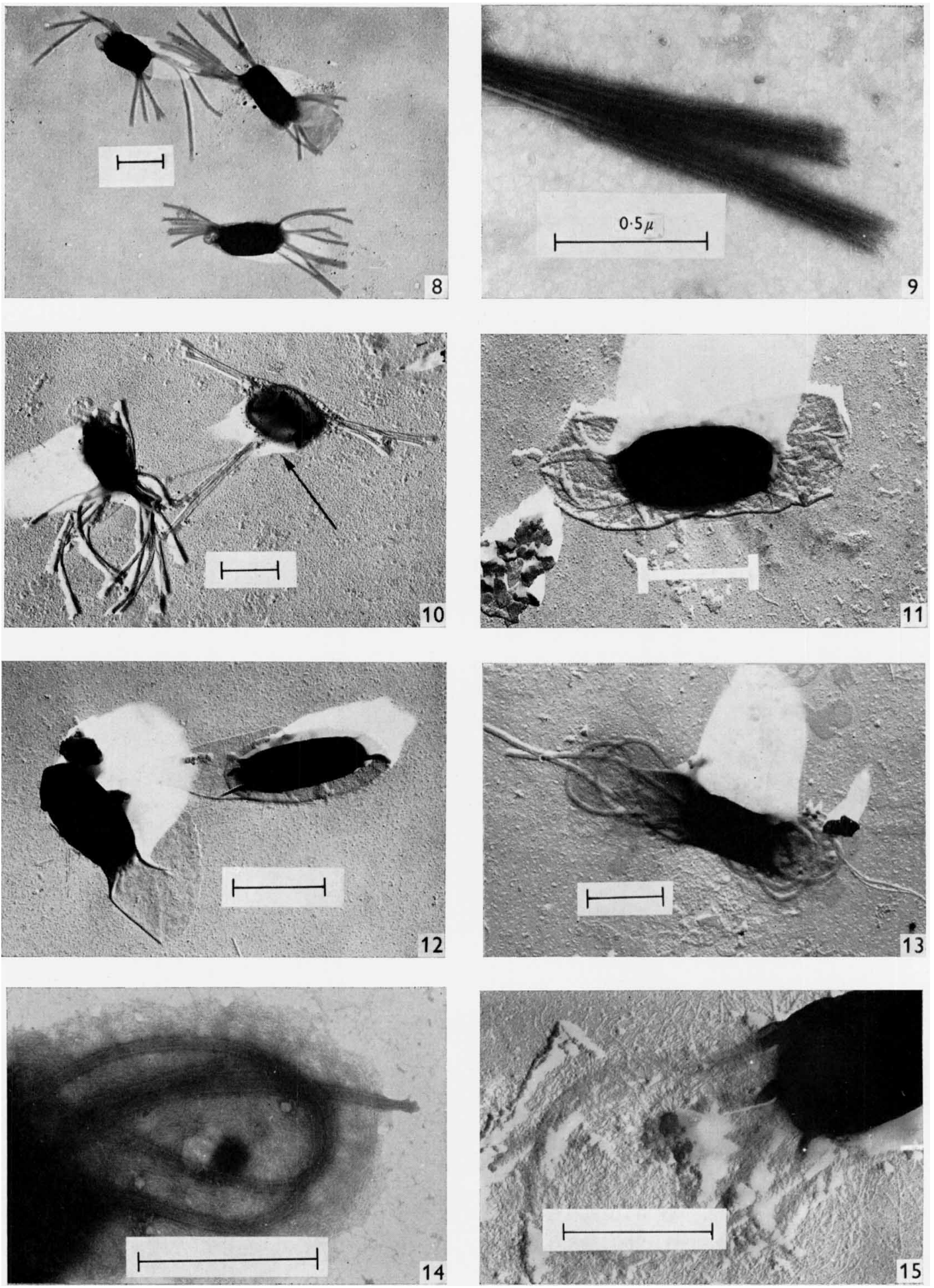

W. HODGKISS, Z. J. ORDAL AND D. C. CANN 

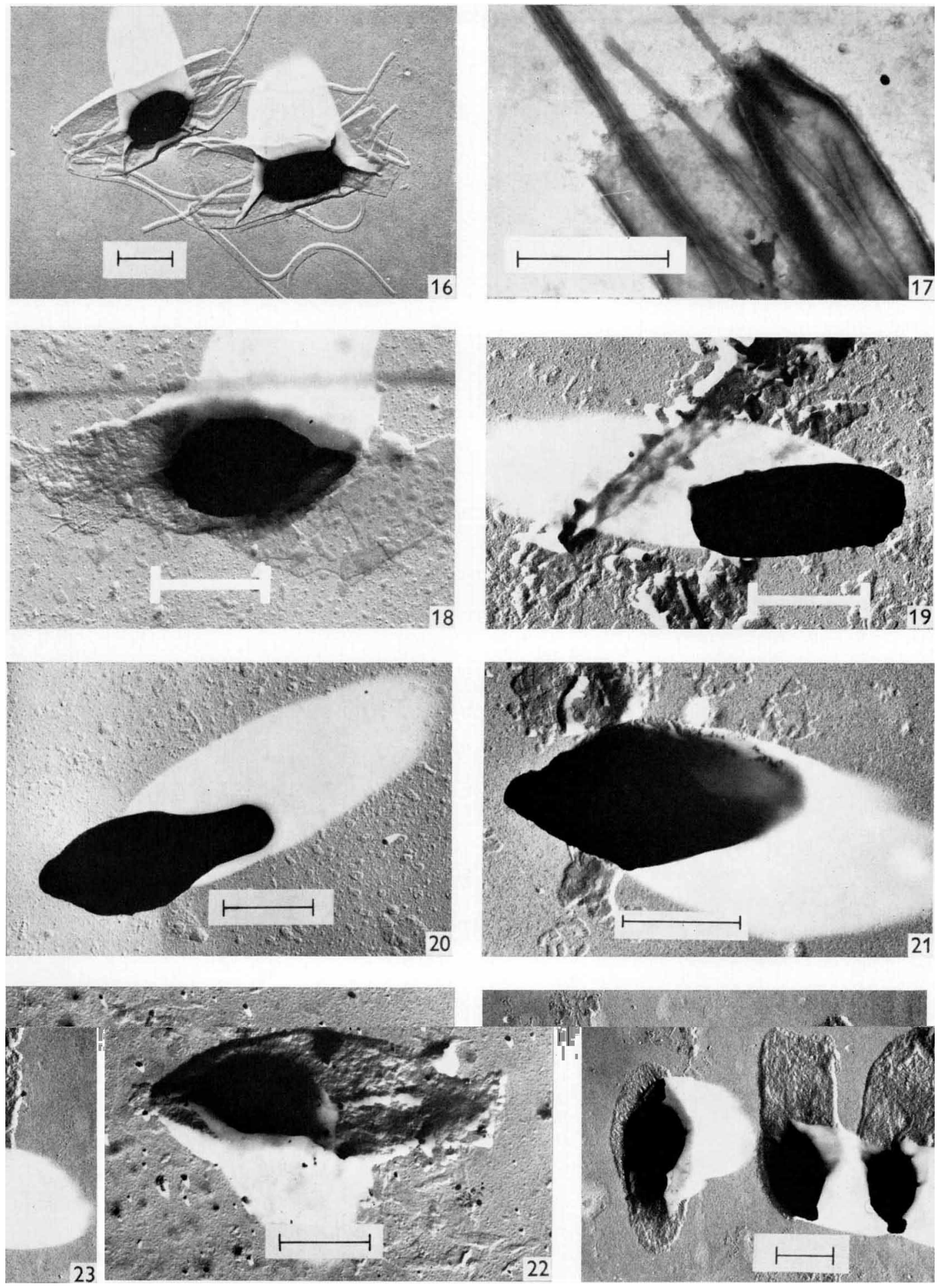

W. HODGKISS, Z. J. ORDAL AND D. C. CANN 

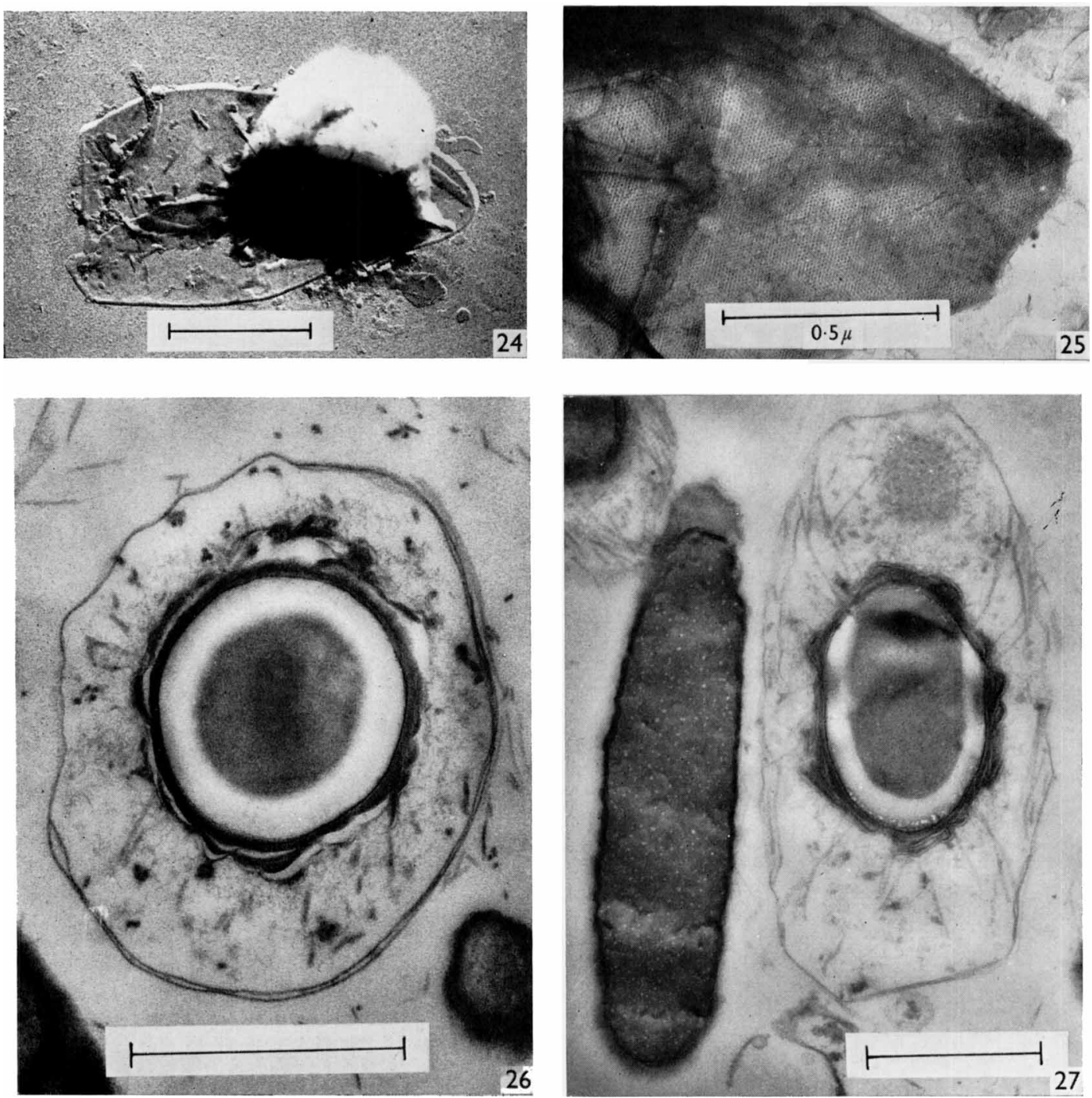


\section{Plate 4}

Fig. 24. C. botulinum type $\mathrm{F}$, strain 610B. Spore and voluminous exosporium.

Fig. 25. C. botulinum type F, strain $610 \mathrm{~B}$. Ammonium molybdate preparation showing the hexagonal pattern structure of the exosporium.

Fig. 26. Section through a mature spore of $C$. botulinum type E, Beluga strain, NCIB4248. Proceeding from the centre of the spore the section shows the dense core, the clear cortex, a thin electron-dense inner spore coat, a multilayered outer spore coat and appendages surrounded by the exosporium.

Fig. 27. C. botulinum type E, Beluga strain, NCIB 4248. Section showing the relative size of a vegetative cell and a mature spore and its exosporium. 